\title{
Los procesos de internacionalización en las pymes: reflexión desde diferentes perspectivas
}

\author{
The internationalization \\ processes in SMEs: reflection \\ from different perspectives
}

\section{Sandra Patricia Rojas Berrío (Colombia) \\ srojasbe@poligran.edu.co \\ Institución Universitaria Politécnico Grancolombiano}

\author{
Ricardo Arturo Vega Rodríguez (Colombia) \\ rvegarod@poli.edu.co \\ Institución Universitaria Politécnico Grancolombiano
}

\begin{abstract}
Resumen
En este artículo se muestran las diferentes perspectivas que se han estudiado sobre la internacionalización de las pymes y el auge que han tenido, ya que los campos de investigación han sido muy fructíferos en esta área.

Se resalta cómo en las últimas décadas se han producido cambios que han transformado definitivamente el entorno económico internacional, los cuales han generado oportunidades para las
\end{abstract}

\begin{abstract}
This article shows the different perspectives that have been studied on the internationalization of SMEs and the boom they have had, since the fields of research have been very successful in this area.

It highlights how in recent decades there have been changes that have transformed definitely the international economic environment, which has created opportunities for companies in foreign markets.
\end{abstract}

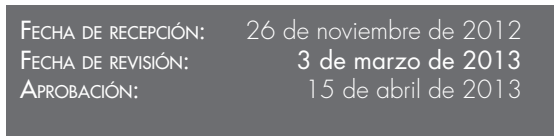

FECHA DE RECEPCIÓN: APROBACIÓN:
3 de marzo de 2013

15 de abril de 2013
Para Citar este artículo / to CIte this article Rojas, S. \& Vega, R. Los procesos de internacionalización en las pymes: reflexión desde las diferentes perspectivas. Poliantea IX, (16), pp. 53-70. 
empresas en mercados extranjeros.

De acuerdo con lo anterior, a medida que pasa el tiempo, se han fomentado distintas iniciativas para incentivar la salida de pequeñas y medianas empresas al exterior. Se revisan y puntualizan las barreras y los facilitadores de la internacionalización destacando el papel que desempeñan las redes en estas empresas para este tipo de procesos.

Palabras clave: pymes,

internacionalización, born global, orientación al mercado.
According to the above, as time passes, various initiatives have been promoted to boost the projection of small and medium enterprises abroad. We review and point out the barriers and facilitators of internationalization highlighting the role of networks in these companies for this type of process.

Keywords: SMEs, internationalization, born global, market orientation. 


\title{
Los procesos de internacionalización en las pymes: reflexión desde diferentes perspectivas
}

\begin{abstract}
Sandra Patricia Rojas Berrío (Colombia)
Doctoranda en Ciencias Administrativas, Instituto Politécnico Nacional, México. Magíster en Administración, Universidad Nacional de Colombia. Administradora de Empresas, Universidad Nacional de Colombia. Directora Departamento Investigación, Desarrollo e Innovación, Institución Universitaria Politécnico Grancolombiano.
\end{abstract}

Ricardo Arturo Vega Rodríguez (Colombia) Magíster en Administración, Universidad Nacional de Colombia. Especialista en DesarroIlo Gerencial, Universidad de los Andes. Químico Farmacéutico, Universidad Nacional de Colombia. Docente investigador, Institución Universitaria Politécnico Grancolombiano.

\section{Introducción}

Las pymes se han constituido en uno de los sectores productivos más importantes para las economías de los países emergentes, debido a su aporte en la dinámica económica. De hecho, su importancia en la economía cada vez se hace más evidente, ya que dentro de la generación de crecimiento económico su impacto directo sobre la creación de empleo y su participación dentro del producto interno bruto (PIB) es significativo y, paradójicamente, al contrario de lo que se pudiera pensar, hay una relación inversa entre la renta de los países y la contribución de las pymes en la generación de riqueza. Se observa que para las naciones de rentas bajas las pymes generan un $31 \%$ del empleo y un $15 \%$ del PIB; para las de rentas medias son alrededor del $55 \%$ de generación de empleo y casi un $40 \%$ del PIB. Y en el caso de las naciones de rentas altas, las pymes logran niveles de alrededor del $65 \%$ en la generación de empleo y representan un $50 \%$ dentro del PIB (Ayyagari, Beck y Demirgüç-Kunt, 2003). 
Teniendo en cuenta la importancia manifiesta de este tipo de compañías en la economía global, se convierte en un tema muy vigente estudiar las prácticas de gestión que desarrollan en sus actividades empresariales. Aunado a las actuales tendencias internacionales de globalización, uno de los elementos que hoy es importante revisar en las pymes es el atinente a sus esfuerzos de llevar sus productos a los mercados globales para, de esta manera, aportar con análisis y reflexiones sobre la manera como podemos mejorar dichos procesos en este tipo de organizaciones.

En Colombia, la Ley 905 de 2004 define las mipymes (micro, pequeñas y medianas empresas) como "Toda unidad de explotación económica, realizada por persona natural o jurídica, en actividades empresariales, agropecuarias, industriales, comerciales o de servicio, rural o urbana, que responda a los siguientes parámetros":

\section{Microempresa. Planta de perso-} nal no superior a los diez (10) trabajadores o activos totales excluida la vivienda por valor inferior a quinientos (500) smlv (salario mínimo legal vigente).
2. Pequeña empresa. Planta de personal entre once (11) y cincuenta (50) trabajadores o activos totales por valor entre quinientos uno (501) y menos de cinco mil (5000) smlv.

3. Mediana empresa. Planta de personal entre cincuenta y uno (51) y doscientos (200) trabajadores o activos totales por valor entre cinco mil uno (5001) y treinta mil (30 000) SMLV.

Por lo tanto, la gran empresa sería aquella cuya planta de personal es mayor a 200 trabajadores o que cuente con activos totales por un valor superior a $30000 \mathrm{smlv}$.

\section{Escuelas sobre procesos de internacionalización}

Como anotan Bell y McNaughton:

La internacionalización de empresas es una actividad de importancia creciente para las economías y para los países, dado que los mercados externos constituyen una alternativa importante de crecimiento y supervivencia en un entorno económico cada vez más competitivo, cuyo proceso es dinámico y que se ha tratado de explicar mediante el uso de modelos teóricos con diversidad de enfoques, los cuales, por lo general, suelen agruparse en dos grandes corrientes: las que siguen el enfoque tradicional (secuencial 
o por etapas) del proceso de internacionalización y las que proponen otros modelos alternativos (2000 p. 67).

Tal como afirman Acedo y Florin (2006), la teoría tradicional de internacionalización, modelo de Uppsala (modelo U), sostiene que la experiencia y los recursos de una empresa en su mercado local facilitan la capacidad para expandirse en el mercado internacional en un proceso que se lleva por etapas. Sería un proceso secuencial que conduciría a la compañía de los mercados domésticos a los internacionales bajo un "proceso de aprendizaje" donde se logra conocimiento de los nuevos mercados y se van comprometiendo gradualmente recursos a estos mercados (Armario, Ruiz y Armario, 2008). Las decisiones de internacionalización son limitadas por dos factores: la información y los recursos disponibles. Consecuentemente, las mayores barreras para este proceso serían la carencia de información y la escasez de recursos. En la tabla 1, se relacionan las variables encontradas en Paunović y Prebežac (2010) respecto de las barreras de internacionalización.

Tabla 1. Variables de las barreras de internacionalziación

\section{BARRERAS A LA INTERNACIONALIZACIÓN}

Carencia de competencias emprendedoras, de gestión y de mercadeo Burocracia

Limitado acceso a información y conocimiento

Carencia de recursos financieros

Baja capacidad para invertir (tecnología, equipos, Know-How)

Discrepancias en cuanto a estandarización, falta de foco a la importancia de la calidad

Diferencias en los usos del producto o servicio

Barreras culturales y de lenguaje

Riesgos de vender en el exterior

Competencia de compañías

Comportamiento inapropiado de las multinacionales con las empresas domésticas

Documentación compleja (empacado y etiquetado)

Carencia de incentivos por parte del Gobierno para la internacionalización

Inadecuados mecanismos de protección intelectual

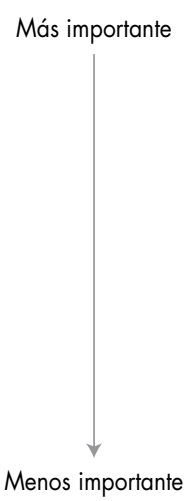

Adaptado de Paunović y Prebežac (2010, p. 61).

Este modelo ha sido criticado por ser determinístico y por prestar poca atención a los contextos particulares (Armario, Ruiz y Armario, 2008). Desde este modelo, la compañía inicia en los mercados domésticos sin tener exportaciones. Después de fortalecerse localmente, la empresa comienza exportaciones a través de agentes de ventas, para 
posteriormente organizar una subsi- de la empresa y aquellas de los otros diaria de ventas en el/los mercado/s miembros de la red de negocios a internacional/es y finalizar con la que pertenece la organización, y una/s subsidiaria/s de manufactura también entre la totalidad de estas en el/los mercado/s foráneo/s. ventajas y las ventajas de localiza-

Pero cada paso del modelo $\mathrm{U}$ se puede considerar como una innovación para la empresa y, por lo mismo, se han propuesto modelos de internacionalización relacionados con la innovación (Ruzzier, Hisrich y Antoncic, 2006; Mejri y Umemoto, 2010). En este caso, se identifican tres estadios genéricos: la fase de preexportación, la fase inicial de exportación y la fase avanzada de exportación.

Como evolución también de la teoría de Uppsala se llega al modelo de redes, en el cual la internacionalización de una compañía está relacionada con un proceso interactivo entre las ventajas competitivas ción de los países (Amal y Rocha, 2010). En este contexto, se define red de negocios como "conjuntos de relaciones de negocios internacionales, en la cual cada relación de intercambio es entre firmas de negocios conceptualizadas como actores colectivos" (Amal y Rocha, 2010, p. 610). Para Karlsen, Silseth, Benito y Welch (2003) el principal supuesto es que los negocios internacionales tienen lugar dentro de un marco de redes con tres variables básicas en juego: actores, recursos y actividades.

Pero dentro de las redes se pueden presentar varios escenarios, que se presentan en la figura 1 : 


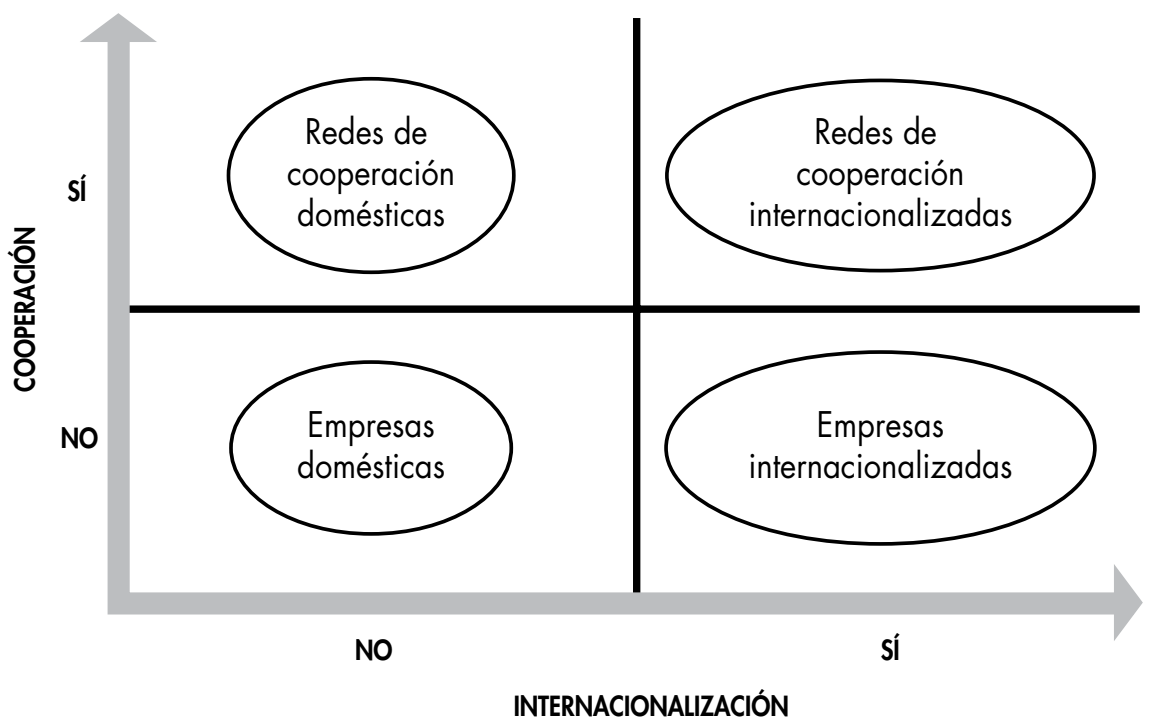

Figura 1. Matriz de cooperación-internacionalización. Tomado de Cal, Verdugo y Fernández-Jardón (2007, p. 7).

En este caso, las empresas nacionales son aquellas que no realizan actividades ni de cooperación ni de internacionalización. Las internacionalizadas hacen el proceso de internacionalización, pero solitarias. Las de cooperación nacionales solo realizan esta asociatividad en el ámbito local y, finalmente, las de cooperación internacionalizadas han alcanzado los mercados internacionales bajo esquemas cooperativos (Cal, Verdugo y Fernández-Jardón, 2007, pp. 6-7). Desafortunadamente, en específico para las pymes colombianas, la asociatividad no ha sido muy fructífera, ya que tienen una cultura empresarial centrada en la organización y con gran desconfianza hacia el exterior y pocas tienen una estrategia de asociación (Calle y Tamayo, 2005).

Comparado con el modelo de Uppsala, encontramos en el otro lado del espectro la teoría de las born global

[...] que son pequeñas y medianas empresas emprendedoras que presentan un proceso de internacionalización acelerado, es decir, inician sus actividades comerciales en el mercado mundial justo apenas nacen o en un periodo muy cercano a su nacimiento (Bell y McNaughton, 2000, p. 142).

A las born global se les reconoce como un fenómeno donde las empresas inician en sus primeros dos años de vida el proceso de 
exportación; para este proceso también se tienen en cuenta la experiencia que deben adquirir a lo largo del tiempo y ciertas etapas de incorporación en las operaciones internacionales. "El fenómeno born global sugiere un replanteamiento de las teorías tradicionales de internacionalización" (Escandón, 2009, p. 57), y en algunos aspectos está inclusive en fuerte oposición a estos modelos tradicionales (Madsen y Servais, 1997). De hecho, Rialp, Rialp y Knight (2005) afirman que esta forma de internacionalización constituye, cada vez más, un patrón distintivo de los procesos de internacionalización de algunas pymes cuando se compara con otro tipo de negocios. Es tan importante el fenómeno del born global que se requieren nuevos marcos de referencia de los procesos de internacionalización y dentro de estos aquellos que expliquen la estrategia de estas firmas, jóvenes y dinámicas, que participan de los mercados mundiales desde su surgimiento (Lozano, Morgan y Robson, 2007).

\section{Facilitadores y barreras de la internacionalización}

Amal y Rocha (2010) encontraron que las políticas públicas no son un factor determinante del desempeño internacional, pero que el involucramiento de instituciones públicas que promuevan la competitividad internacional de las compañías sí lo es en el inicio de estos procesos. Complementan afirmando que los capitales para el emprendimiento, las estrategias de toma de riesgos, la habilidad para cooperar y aprender a través de redes son fuerzas que sí son determinantes para una internacionalización rápida y sostenible. En contraste, Wright, Westhead y Ucbasaran (2007) afirman que las empresas deberían experimentar "épocas" de internacionalización, por lo que las políticas públicas deberían considerar esta dinámica como un proceso integral en el tiempo más que verla como una sola actividad de despegue.

De hecho, y en relación con las redes, Chetty y Stangl (2010) relacionan seis beneficios que se derivan de estas, como son: distribución de los riesgos, acceso a nuevos mercados y tecnologías, rapidez en la comercialización, acumulación de activos complementarios, protección de derechos de propiedad y el papel que desempeñan las redes, como el acceso a conocimiento externo. Lo que lleva a la importancia del tipo de 
relaciones en las redes y cómo son usadas por las firmas para innovar e internacionalizarse. Complementan este concepto Musteen, Francis y Datta (2010) al encontrar que los resultados de la expansión rápida en los mercados foráneos dependen de las capacidades que las empresas desarrollen en las etapas iniciales de la internacionalización. Dentro de este concepto surge lo hallado por Manolova, Manev y Gyoshev (2010) en el sentido de que el papel que desempeñan las redes cambia en el tiempo, lo que sugiere que las asociaciones y conexiones establecidas tempranamente en la vida de la nueva firma son más productivas para su internacionalización.

Refuerza la importancia de las redes el hecho destacado por Prashantham (2011) en cuanto un ambiente regional desfavorable, en una economía emergente, podría reforzar las restricciones de recursos pero, al mismo tiempo, permitiría que el papel de las redes descollara más fuertemente. Portugal, Carvalho y Ribeiro (2010) añaden que la restricción de recursos lleva a que las firmas se enfoquen en nichos de mercado en el extranjero y que el despliegue de sus redes de relaciones haría más fácil la adaptación a las idiosincracias de los nichos de mercado seleccionados en el exterior.

Cancino y La Paz (2010) incluyen como factores causales de una rápida internacionalización los relacionados con las características del fundador, las capacidades organizacionales y el foco estratégico de la firma. En el primer punto, destacan la visión global del fundador de la compañía y su experiencia internacional. Respecto del segundo punto, se relacionan la capacidad de aprendizaje en cuanto a los mercados externos, los activos intangibles con que cuente la empresa y el hecho de participar en sectores de mayor nivel tecnológico. Y en el último factor aparecen el deseo de posicionarse en nichos de mercado específicos, mientras que las distancias geográficas no son vistas como barreras de internacionalización.

Escolano y Belso (2003) mencionan que parte de la existencia de imperfecciones en los mercados, sobre todo, en los productos intermedios, es lo que llevará a una progresiva internacionalización de las transacciones de dichos productos. Según Buckley y Casson (2003) existen cuatro grupos de factores relevantes para adoptar decisiones de internacionalización de mercados: 
1. Factores de la industria: tipo de producto y mercado.

2. Factores de la región: rasgos geográficos y sociales vinculables al mercado.

3. Factores de la nación: relaciones políticas y fiscales internacionales.

4. Factores de la empresa: habilidades de la dirección a la hora de internacionalizar.

El objetivo de algunas investigaciones se basó en el análisis de la internacionalización de los proveedores y a la vez en qué medida es condicionada por la relación proveedor (pymes)-cliente (empresas multinacionales). De esta manera, se divisó un grupo de situaciones donde se "obtuviera algunos indicios sobre la problemática de la internacionalización en las empresas industriales en la relación proveedor-cliente" (Moreira, 2007, p. 103).

De acuerdo con este autor, el proceso de internacionalización depende de las siguientes dimensiones:

1. productos y actividades;

2. diversos modos de entrada y operación;

3. mercados;

4. competencias internas;
5. capacidad de administrar relaciones cooperativas;

6. aspectos financieros;

7. estructura organizacional.

Se pueden, entonces, distinguir dos corrientes principales para explicar el proceso de internacionalización: la primera defiende un modelo evolutivo, secuencial y lineal con participaciones internacionales crecientes, y la segunda observa el proceso de internacionalización desde el punto de vista de los recursos y de la interacción con el entorno ambiental.

Rodríguez (2007), además, establece diferencias entre los factores locales e internacionales que estimulan los procesos de internacionalización de las grandes compañías, comparadas con las pymes. Así, los procesos de internacionalización de las empresas grandes se pueden iniciar con la búsqueda de proveedores de partes y materiales, pero, igualmente, se puede dar en el afán de asegurar mercados para sus propios productos. En contraste, las pymes podrían comenzar estos procesos debido a factores, como la disminución de la participación en el mercado local, el aumento de la competencia nacional, para obtener 
economías de escala, por crecimiento de los mercados externos o por el surgimiento de un cliente importante en el exterior. Se añade que debido a la disponibilidad de recursos es más común que las grandes compañías monten fábricas en el exterior que las pymes y en ambos casos también se recurre a la figura de la franquicia.

\section{Orientación al mercado y potencial de internacionalización}

Se ha estudiado igualmente la relación que puede existir entre la orientación al mercado de las organizaciones y su potencial de internacionalización. Jaworski y Kohli (1993) plantean un modelo de orientación al mercado que propone, además, una serie de antecedentes que influenciarían el nivel de orientación al mercado de una empresa y de consecuencias tanto en los empleados como en el desempeño del negocio. Asimismo, incluyen una serie de factores moderadores del ambiente que también influirían en la orientación al mercado de una compañía. Este modelo es presentado en la figura 2.

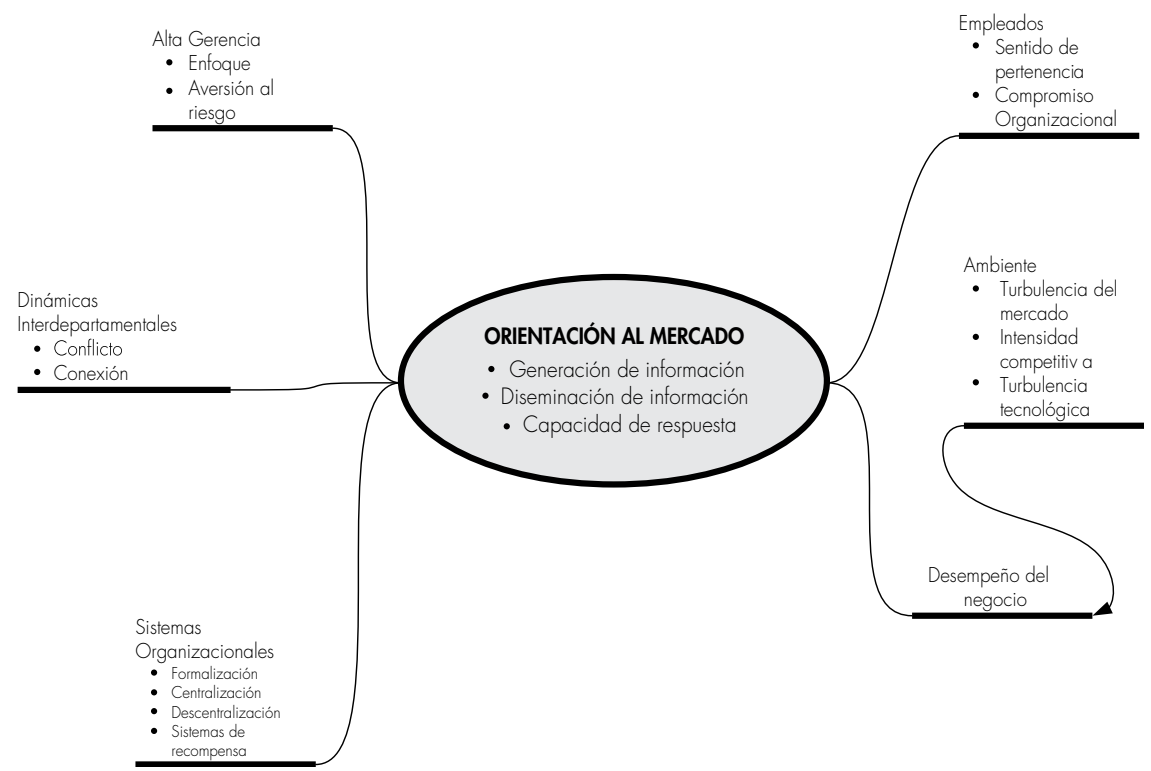

Figura 2. Orientación al mercado.

Adaptado de Jaworski y Kohli (1993, p. 55). 
Este modelo se centra en la gestión de la información dentro de la firma, en sus componentes de consecución, dispersión interna y utilización posterior. Considera que la información se convierte en el eje fundamental sobre el cual la organización debe centrar sus actividades, que la lleven a lograr una posición competitiva fuerte en su entorno sectorial.

Zheng, Brown, Dev y Agarwal (2007) analizaron qué tanto los componentes de orientación al cliente y a los competidores, dentro de la orientación al mercado de una firma, deben ser adaptados de acuerdo con los aspectos ambientales de la organización. Sus resultados indican que privilegiar la orientación al cliente es mejor en mercados desarrollados económicamente, al igual que en mercados con buenas condiciones locales de negocios, mayor disponibilidad de recursos y cuando los clientes son demandantes. En contraste, la orientación a los competidores funciona mejor en mercados económicamente en desarrollo, que tengan condiciones locales de negocios pobres y que enfrenten escasez de recursos.

\section{Relaciones interorganizacionales}

En cuanto a la importancia de la relación interorganizacional en la cadena de suministros, se concluyó que los factores más importantes que afectan la relación proveedorcliente son la tecnología acumulada, la estructura y el tamaño de la empresa. Es necesario tener en cuenta que para mantener relaciones internacionales estables no es recomendable poseer un elevado número de proveedores.

"Wheelwright \& Clark (1995) demostraron que las competencias de desarrollo cooperativo de nuevos productos (DCNP) son cruciales en las interrelaciones" (Moreira, 2007, p. 95).

Avanzando en la investigación se distinguieron dos tipos de relación proveedor-cliente: relaciones de mercado a largo plazo y alianzas estratégicas.

De acuerdo con ciertos apartados, algunos de los beneficios de las relaciones proveedor-cliente son:

1. Un proveedor puede fácilmente acceder a la tecnología del socio.

2. Se generan ventajas competitivas a largo plazo. 
3. Se generan mejoras en la productividad de corto plazo.

4. Originan menores costos en investigación y desarrollo, producción y distribución.

\section{Cambio organizacional}

Es importante empezar los procesos de internacionalización desde un enfoque hacia el cambio organizacional, frente al continuo y rápido cambio mundial, ya que la mejor estrategia es la adaptación; sin embargo, se debe tener en cuenta que el tiempo para dicha adaptación cada vez es más corto.

"Cambio organizacional puede definirse como la capacidad de adaptación de las organizaciones a las diferentes transformaciones que sufran sus ambientes interno o externo" (Calle y Tamayo, 2005, p. 139).

Estos autores nombran uno de los modelos más conocidos sobre el proceso del cambio, el cual es presentado por Lewin (1988), donde asegura que para tener éxito en el cambio es necesario descongelar el statu quo, realizar una transición a un nuevo estado y luego recongelar este último para que el cambio sea permanente.

Teniendo en cuenta los cambios constantes mundiales en las organizaciones y sus necesidades de adaptación, Senge (1997) introdujo el concepto de organizaciones que aprenden (learning organizations).

Según Calle y Tamayo (2005, p. 140), las opciones de cambio en una empresa se dividen en tres categorías: estructura, técnicas y tecnología-personas.

La clasificación de las personas en un proceso de cambio es indispensable por tal motivo. Strebel (1997, p. 140) determinó las siguientes categorías de participantes: agentes de cambio, opositores, espectadores y tradicionalistas.

Otro punto básico es la competitividad en el ámbito de las naciones, y para lo cual Strebel (1997) habla de una competitividad sistémica, que tendrá su perspectiva desde cuatro niveles: metanivel, macronivel, mesonivel y micronivel. Dicho concepto establece que si existe alguna deficiencia en uno de estos niveles, es sinónimo de baja competitividad y subdesarrollo.

Tal como lo menciona Porter (1991, p. 142), los cuatro atributos genéricos en una nación pueden ser determinantes de su éxito en cierto sector: condiciones de los factores, condiciones de la demanda, sectores afines/apoyo y estrategia, estructura y rivalidad de las empresas. 
En la competitividad en el ámbito de las naciones, es importante puntualizar las diferencias entre las ventajas comparativa y competitiva, tal como lo anota Enrique Martínez Moreno de la Universidad Autónoma Metropolitana de México (citado por Calle y Tamayo, 2005, p. 145) cuando afirma:

Se parte de la idea de que las empresas y no las naciones son las que compiten en los mercados internacionales. Realmente pocas empresas han logrado insertarse adecuadamente en la dinámica del comercio mundial, de manera que en muchas ocasiones se han formado grandes grupos empresariales $o$ bloques económicos que han aumentado la brecha entre sectores y entre países (p. 144).

Las empresas deberán tener ventajas competitivas, y estas podrán presentarse de dos maneras: costos inferiores y diferenciación.

La decisión de una empresa de internacionalizarse puede determinarse por cuatro razones generales: incremento de las ventas, adquisición de recursos, diversificación de sus fuentes de ventas y suministros y reducción del riesgo competitivo. Existen cinco etapas de desarrollo en los mercados internacionales:
1. desarrollo internacional;

2. desarrollo comercial;

3. desarrollo contractual;

4. desarrollo participativo;

5. desarrollo integrado y autónomo (Calle y Tamayo, 2005, p. 149).

Avella y López (1995) establecen cuatro tipos de estrategias para incorporarse en los mercados internacionales, estas son:

1. estrategia global;

2. estrategia regional;

3. estrategia internacional;

4. estrategia multilocal.

\section{Conclusiones}

1) Dada la importancia de las pymes en la economía colombiana, es fundamental lograr que estas empresas incorporen en sus prácticas gerenciales elementos que les permitan ser competitivas no solo en los mercados locales, sino también en los internacionales, teniendo en cuenta la significación que pudieran llegar a tener en las exportaciones del país, especialmente, aquellas no tradicionales.

2) Un elemento fundamental para el logro de esta competitividad 
internacional es adquirir habilidades de aprendizaje organizacionales, lo que va a permitir que las empresas se puedan desarrollar como empresas flexibles, dinámicas $\mathrm{y}$, de esta manera, se puedan adaptar a las condiciones cambiantes de los mercados globales.

3) Pero el aprendizaje para la internacionalización no solo es un proceso que incentiva la presencia en el exterior de las empresas, sino que debe propender a que las compañías estén mejor preparadas en sus esfuerzos por competir en los mercados locales con firmas extranjeras, que cada vez más aumentan las alternativas de consumo para la población local, incentivadas, a su vez, por la proliferación de los tratados de libre comercio entre diferentes países y bloques económicos de todas partes del mundo.

4) En muchos de los estudios y de los contextos estudiados surge recurrentemente la asociatividad como uno de los factores clave para el logro de la competitividad internacional, que es un hecho particularmente importante para las pymes dado el acceso más limitado que tienen a los recursos cuando se las compara con las grandes empresas. En Colombia, se han realizado algunos esfuerzos persistentes para desarrollar esquemas asociativos, tipo Federación Nacional de Cafeteros, pero falta mucho camino por recorrer para que, efectivamente, la asociatividad se convierta en uno de los elementos estratégicos prioritarios de las organizaciones colombianas, ya que predomina la cultura individualista.

Las born global se están convirtiendo en una proporción muy importante de las empresas exportadoras en el mundo entero. Denota cómo cada vez más las firmas deben fortalecer su dinamismo y agilidad en sus esfuerzos internacionales, ya que la competencia global lo exige y presupone un esfuerzo mucho más focalizado de los estados y gremios para generar sustratos industriales y de negocios que les faciliten a estas compañías su rápido despegue hacia los mercados foráneos.

\section{Referencias}

Acedo, F. y Florin, J. (2006). An entrepreneurial cognition perspective on the internationalization of SMEs. Journal of International Entrepreneurship, 4, 49-67. 
Amal, M. y Rocha, A. (2010). Internationalization of small-and medium-sized enterprises: a multi case study. European Business Review, 22(6), 608-623.

Armario, J.; Ruiz, D. y Armario, E. (2008). Market orientation and internationalization in small and medium-sized enterprises. Journal of Small Business Management, 46(4), 485-511.

Avella Camarero, L. y López Duarte, C. (1995). Alternativas estratégicas para la internalización de un negocio. Alta Dirección, 183, 49-58.

Ayyagari, M.; Beck, T. y Demirgüç-Kunt, A. (2003). Small and medium enterprises across the globe. Policy Research Working Paper, The World Bank, Development Research Group Finance, Washington.

Bell, J. y McNaughton, R. (2000). "Born global" firms: a challenge to public policy in support of internationalization. Marketing in Global Economy, 176-185.

Buckley, P. y Casson, M. (2003). The future of the multinational enterprise in retrospect and in prospect. Journal of International Business Studies, 34, 219-222.
Cal, M.; Verdugo, M. y FernándezJardón, C. (2007). Internacionalización-cooperación en las pymes de Vigo y de su área metropolitana. Revista Galega de Economía, 16(1), 1-13.

Calle, A. y Tamayo, V. (2005). Estrategia e internacionalización en las pymes: caso Antioquia. Cuadernos de Administración, 18(30), 137-164.

Cancino, C. y La Paz, A. (2010). International New Ventures en Chile: tres casos de éxito. Revista Latinoamericana de Administración, 45, 140-162.

Chetty, S. y Stangl, L. (2010). Internationalization and innovation in a network relationship context. European Journal of Marketing, 44(1112), 1725-1743.

Escandón, D. (2009). Factores que inciden en la creación de Born Global en Colombia. Estudios Gerenciales, 25(113), 55-73.

Escolano, C. y Belso, J. (2003). Internacionalización y pymes: conclusiones para la actuación pública a partir de un análisis multivariante. Revista Asturiana de Economía, 27, 169-195. 
Jaworski, B. y Kohli, A. (1993, julio). Market orientation: antecedents and consequences. Journal of Marketing, 57, 53-70.

Karlsen, T.; Silseth, P.; Benito, G. y Welch, L. (2003). Knowledge, internationalization of the firm, and inward-outward connections. Industrial Marketing Management, 32, 385-396.

Kurt, L. (1988). La teoría de campo en la ciencia social. Madrid: Paidós.

Lozano, S.; Morgan, R. y Robson, M. (2007). The born global phenomenon in Mexico: a bright start for technology intensive start-ups. Revista del Centro de Investigación. Universidad La Salle. México, 7(028), 111-122.

Madsen, T. y Servais, P. (1997). The internationalization of born globals: an evolutionary process? International Business Review, 6(6), 561-583.

Manolova, T.; Manev, I. y Gyoshev, B. (2010). In good company: the role of personal and inter-firm networks for new-venture internationalization in a transition economy. Journal of World Business, 45, 257-265.
Mejri, K. y Umemoto, K. (2010). Small-and medium-sized enterprise internationalization: towards the knowledge-based model. Journal of International Entrepreneurship, 8, 156-167.

Moreira, A. (2007). La internacionalización de pymes industriales a través de multinacionales. Presentación de algunos casos de los sectores automotor y electrónico. Cuadernos de Administracion, 20(34), 89-114.

Musteen, M.; Francis, J. y Datta, D. (2010). The influence of international networks on internationalization speed and performance: a study of Czech SMEs. Journal of World Business, 45, 197-205.

Paunović, Z. y Prebežac, D. (2010). Internationalization of small and medium-sized enterprises. Tržište/ Market, XXII(1), 57-76.

Porter, M. (1991). La ventaja competitiva de las naciones. Buenos Aires: Vergara.

Portugal, M.; Carvalho, J. y Ribeiro, F. (2010). The international entrepreneurial firms' social networks. Cadernos EBAPE.BR, 8(1), 133-145. 
Prashantham, S. (2011). Social capi- Senge, P. (1997). The fifth discipline. tal and indian micromultinationals. Measuring Bussiness Excellence, British Journal of Management, 22, 1(3), 46-51.

4-20.

Strebel, P. (1997). La política del Rialp, A.; Rialp, J. y Knight, G. cambio: retos y misión de los líderes. (2005). The phenomenon of ear- Capital Humano, 10(101), 24-28. ly internationalizing firms: what do we know after a decade (19932003) of scientific inquiry? International Business Review, 14, 147-166.

Rodríguez, J. (2007). The Internationalisation of the small and medium-sized firm. Prometheus, 25(3), 305-317.

Ruzzier, M.; Hisrich, R. y Antoncic, Wright, M.; Westhead, P. y Ucbasaran, D. (2007). Internationalization of small and medium-sized enterprises (SMEs) and international entrepreneurship: a critique and policy implications. Regional Studies, 41, 1013-1029.

Zheng, K.; Brown, J.; Dev, C. y Agarwal, S. (2007). The effects of customer B. (2006). SME internationalization research: past, present, and future. Journal of Small Business and Enterprise Development, 13(4), 476-497. and competitor orientations on performance in global markets: a contingency analysis. Journal of International Business Studies, 38, 303-319. 